\title{
Performance assessment of a small-size ocean wave energy harvester
}

\author{
Yongjie Sang ${ }^{1}$, Bertrand Dubus ${ }^{2, *}$ \\ ${ }^{1}$ Acoustic Science and Technology Laboratory, Harbin Engineering University, Harbin 150001, China \\ ${ }^{2}$ IEMN UMR8520, Université de Lille, CNRS, Ecole Centrale de Lille, ISEN, Université de Valenciennes, Lille, France
}

\begin{abstract}
A lightweight electromechanical device is studied to harvest energy of ocean waves and supply electrical power to small-size ocean observation equipment such as sonobuoys. It is composed of a magnet fixed to the floating housing which follows the motion of the ocean surface and a moving coil connected to the case via a flexible spring. As the floating housing follows the vertical motion of water surface, a voltage is induced in the coil due to relative velocity between the coil and the magnet, and kinetic energy of the ocean wave is converted into electrical energy. Full bridge rectifying circuit and smoothing capacitor are used to convert AC voltage to constant voltage. Single degree of freedom electromechanical model of the prototype transducer (LGT-4.5 geophone) is developed and simulated with an electrical circuit software to predict energy harvesting performance. Vibration experiments are also performed with a shaker to validate transducer model and quantify output voltage. Parametric analysis is conducted to identify optimal choice of capacitance in terms of maximum stored energy and minimum charging time. This device is simple and small size relative to ocean wavelength compared to classical linear permanent magnetic generator used in offshore power plant. Its power generation per unit weight is compared to larger scale ocean energy converters.
\end{abstract}

\section{Introduction}

Research on energy harvesting of ocean waves mainly focuses on large-scale hydraulic system to provide power in the $\mathrm{kW}$ range [1,2]. Ocean sonobuoys or portable emergency rescue devices may also benefit from small-scale harvester with limited power capability. However, contrary to large-scale systems having size and time constant comparable to ocean wavelength and period, new concepts have to be developed for miniaturized oceanic energy harvesters. In this work, a device is studied to convert the kinetic energy in the vertical direction of a floating object into electrical energy using a moving-coil transducer.

\section{Ocean wave theory}

Wind waves are surface waves in the ocean. They are generated by the mechanical force of the wind acting on the free surface. For frequencies larger than $0.05 \mathrm{~Hz}$ and small amplitude conditions, the water surface particle undergoes a sinusoidal motion under the action of gravity and surface tension (Fig.1)

$$
u=\frac{H}{2} \sin (k x-\omega t)
$$

where $\lambda$ is wave length, $H$ is wave height, $h$ is depth of water and $x$ is direction of wave motion.

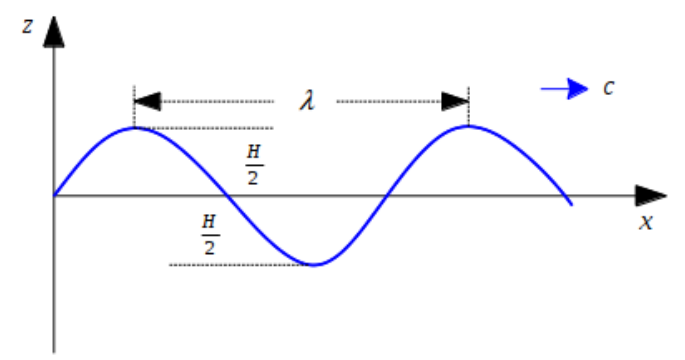

Fig. 1. Waveforms in micro-scale theory [3].

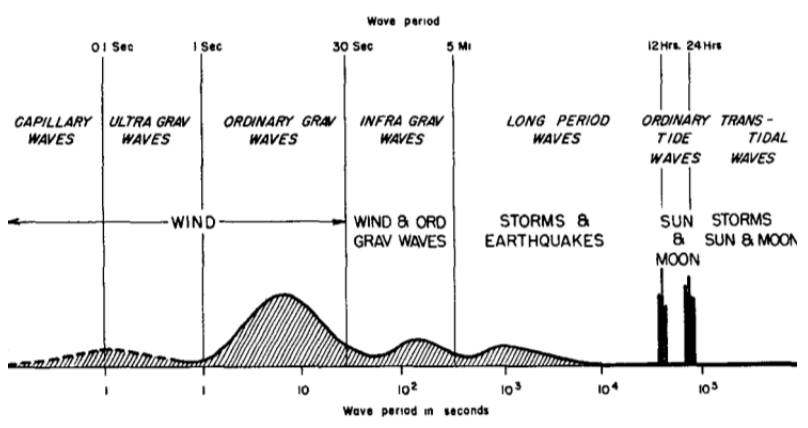

Fig. 2. Ocean wave energy distribution versus frequency [4].

The distribution of ocean wave energy with frequency is displayed in Fig.2. A high energy density is associated with ordinary gravity waves between 0.05 and $1 \mathrm{~Hz}$. However, transduction efficiency from mechanical to electrical energy is expected be low for a small-scale device with a resonance frequency well above this

* Corresponding author: bertrand.dubus@isen.fr 
frequency range. Therefore, the $1-10 \mathrm{~Hz}$ frequency range which is associated to a lower but reasonable energy density is considered in this work. Above $10 \mathrm{~Hz}$, velocity of surface particle is reported to always exceed 0.23 m.s-1 [4]. Moving coil devices are evaluated hereafter for this application as they may exhibit resonant frequencies as low as a few $\mathrm{Hz}$.

\section{Models of the moving coil transducer}

\subsection{Linear analytical model}

The vibration model of the moving coil energy harvester can be regarded as a single-degree-of-freedom massspring-damping system, as shown in Fig.3. The housing is assumed to be floating at ocean surface and therefore to have a given sinusoidal motion of amplitude $u=H / 2$ in the vertical direction. The magnet is fixed to the housing. The relative motion of the coil with respect to the magnet is affected by the spring restoring force, the mechanical friction force and Laplace force. Assuming that time dependence is as $e^{+j \omega t}$, Newton's law is therefore written as

$$
-\omega^{2} m\left(u-u_{1}\right)=k u_{1}+j \omega r_{m} u_{1}+B l i
$$

where $u$ is the displacement of the housing/magnet, $u_{1}$ the displacement of the coil, $m$ the inertial mass (coil), $k$ the stiffness of the flexible spring, $r_{m}$ the damping coefficient of the spring, $B$ magnetic induction in the air gap of the magnetic circuit, $l$ the total length of the coil wire. The electric current $i$ in the coil is expressed using Faraday's law of induction and Kirchhoff's circuit law

$$
\begin{gathered}
e=-j \omega B l u_{1} \\
e+\left(R_{\text {coil }}+j \omega L_{\text {coil }}+Z_{\text {load }}\right) i=0
\end{gathered}
$$

where $R_{\text {coil }}$ is the resistance of the coil, $L_{\text {coil }}$ is the inductance of the coil and $Z_{\text {load }}$ the impedance of the load. Equation (2) may be rewritten as

$$
-\omega^{2} m\left(u-u_{1}\right)=\left[k+j \omega r_{m}+\frac{j \omega(B l)^{2}}{\left(R_{\text {coil }}+j \omega L_{\text {coil }}+Z_{\text {load }}\right)}\right] u_{1}
$$

where $B l$ is the electromechanical conversion factor. Finally, the displacement relationship between the coil and the housing is obtained.

$$
\frac{u_{1}}{u}=\frac{-\omega^{2} m}{k+j \omega r_{m}-\omega^{2} m+\frac{j \omega(B l)^{2}}{\left(R_{\text {coil }}+j \omega L_{\text {coil }}+Z_{\text {load }}\right)}}
$$

Assuming that the load is resistive $\left(Z_{\text {load }}=R_{\text {load }}\right)$ and the inductance of the coil may be neglected, equation (6) is written as [5]

$$
\frac{u_{1}}{u}=\frac{-\left(\omega / \omega_{0}\right)^{2}}{1-\left(\omega / \omega_{0}\right)^{2}+2 j \xi\left(\omega / \omega_{0}\right)}
$$

where $\omega_{0}=\sqrt{k / m}$ is the resonant frequency of the system and $\xi=r /\left(2 \omega_{0} m\right)=r /(2 \sqrt{\mathrm{km}})$ is damping ratio. $r=r_{m}+r_{e}$ is the total damping coefficient where
$r_{e}=(B l)^{2} /\left(R_{\text {coil }}+R_{\text {load }}\right)$ is the contribution of the electric circuit to the mechanical damping. The induced voltage on the coil $V$ is deduced from equation (3)

$$
\frac{V}{u}=\frac{j B l \omega\left(\omega / \omega_{0}\right)^{2}}{1-\left(\omega / \omega_{0}\right)^{2}+2 j \xi\left(\omega / \omega_{0}\right)}
$$

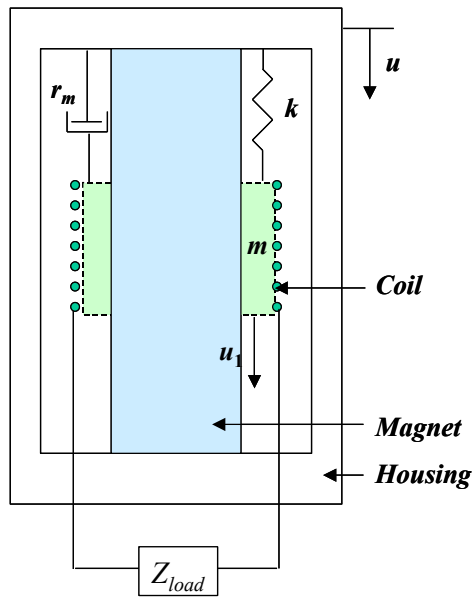

Fig. 3. Schematic diagram of the moving coil harvester.

\subsection{Linear lumped circuit models}

Electromechanical analogies are classically used to describe electroacoustic devices such as microphones, loudspeakers or piezoelectric transducers $[6,7]$. When using an admittance analogy for the mechanical part, the moving coil transducer may be represented by the electrical circuit of Fig.4 [7] where $V_{1}=j \omega u, C_{1}=m$, $L_{1}=1 / k, R_{2}=1 / r, L_{2}=L_{\text {coil }}$ and $R_{3}=R_{\text {coil }}$. One-way conversion from mechanical to electrical energy is described by a voltage source of coefficient $B l$ controlled by a voltage (equal to $j \omega u_{1}$ ). Two limitations should be noted for this model: 1) the mechanical damping of the transducer depends on load resistance and therefore, the model is not really representative of the isolated transducer; 2) strictly speaking, the model is not valid in the case of nonlinear electric loads because $R_{\text {load }}$ can not be defined.

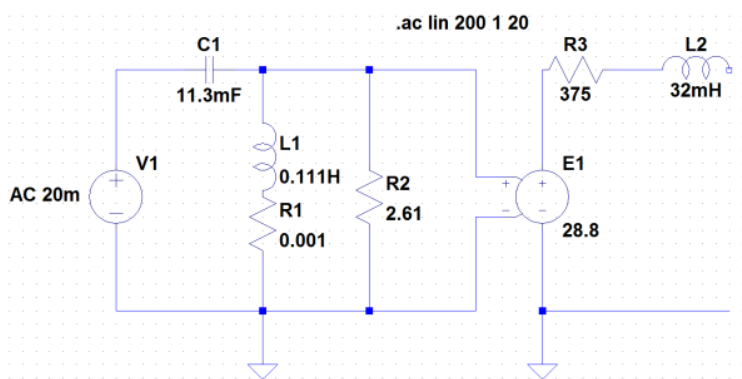

Fig. 4. Electrical circuit model of the moving coil transducer valid for linear loads.

\section{Energy harvesting simulation and measurement}

\subsection{Geophone and experimental setup}


The LGT4.5 seismic geophone manufactured by Longet Inc. (Fig.5) is chosen for the experiments because it resonates at low frequency $[5,8]$. Its main parameters are given in Table 1. For the experiments, the geophone is mounted on a shaker which provides the forced vibration. The vibration amplitude and phase of the shaker are monitored with an accelerometer.

Table 1. Parameters of LGT4.5 seismic geophone.

\begin{tabular}{|c|c|}
\hline Resonance frequency & $4.5 \mathrm{~Hz}$ \\
\hline Inertial mass & $11,310^{-3} \mathrm{~kg}$ \\
\hline Open circuit damping ratio & 0.6 \\
\hline Maximum displacement & $210^{-3} \mathrm{~m}$ \\
\hline Sensitivity & $28.8 \mathrm{~V} \mathrm{~m}^{-1} \mathrm{~s}^{-1}$ \\
\hline Coil inductance & $3210^{-3} \mathrm{H}$ \\
\hline Coil resistance & $375 \Omega$ \\
\hline $\begin{array}{c}\text { Leaf } \\
\text { Spring Housing Cylinder } \\
\text { Coil }\end{array}$ \\
\hline
\end{tabular}

Fig. 5. LGT 4.5 geophone.

\subsection{Geophone sensitivity in open-circuit}

Fig. 6 compares the measured sensitivity (output voltage per unit input velocity) of the geophone to the simulations performed with the analytical and the linear electric circuit model. A very good agreement is obtained in the $2-20 \mathrm{~Hz}$ frequency range. Sensitivity increases linearly below geophone resonance and then stabilizes at $28.8 \mathrm{~V} \mathrm{~m}^{-1} \mathrm{~s}^{-1}$ above $7 \mathrm{~Hz}$.

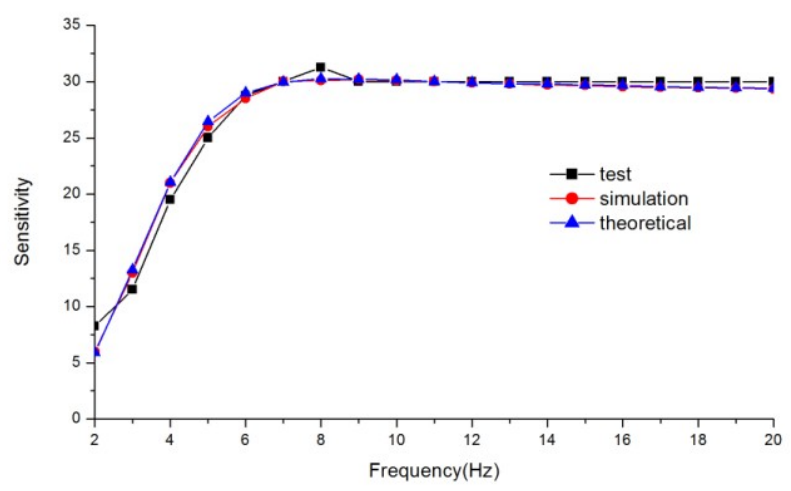

Fig. 6. Measured and simulated sensitivity of the geophone in open circuit. Measurement: black line, analytical model: blue line, circuit model: red line.

\subsection{Energy harvesting with resistive load}

The configuration of the geophone with a resistive load is considered to evaluate the available electrical power for immediate use (without energy storage). The power dissipated in the load versus load resistance is displayed in Fig. 7 for an input vibration velocity of $0.05 \mathrm{~m} . \mathrm{s}^{-1}$ at
$5 \mathrm{~Hz}$. This case corresponds to a frame displacement of $1.59 \mathrm{~mm}$ lower than geophone maximum displacement. As previously reported in [8], it is observed the dissipated power may be maximized if the value of the load resistance is optimized. A maximum dissipated power of $0.109 \mathrm{~mW}$ is obtained for a resistive load of $3 \mathrm{k} \Omega$ and a maximum displacement of the magnet of $1 \mathrm{~mm}$. In Fig.8, this result is compared to existing larger scale device used for ocean energy harvesting in terms of power per unit volume parameter. Hydraulic harvesting devices floating at the surface develop for offshore power plants correspond to the large volumes and masses. The medium power device is a flexural vertical beam clamped at ocean bottom surface which generates electricity via piezoelectric patches [9]. Compared to these higher power systems, the power per unit volume ratio of the geophone-based harvesting device is slightly lower than expected using scaling laws. This may be due to the limitation on the displacement amplitude of the geophone which decreases the useful velocity amplitude to approximately $20 \%$ of ocean surface amplitude and therefore reduces the dissipated power by a factor of 25 .

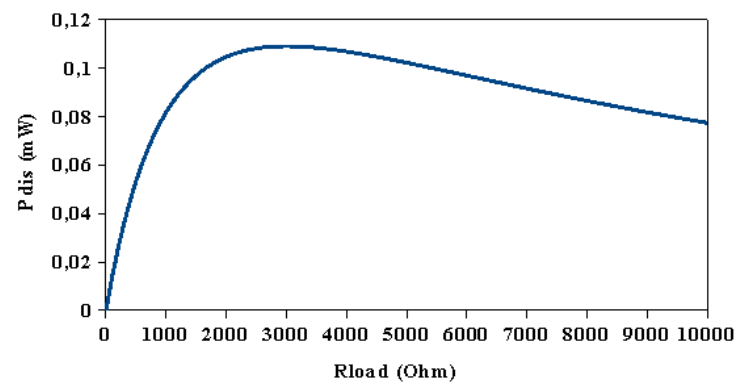

Fig. 7. Dissipated power versus resistive load

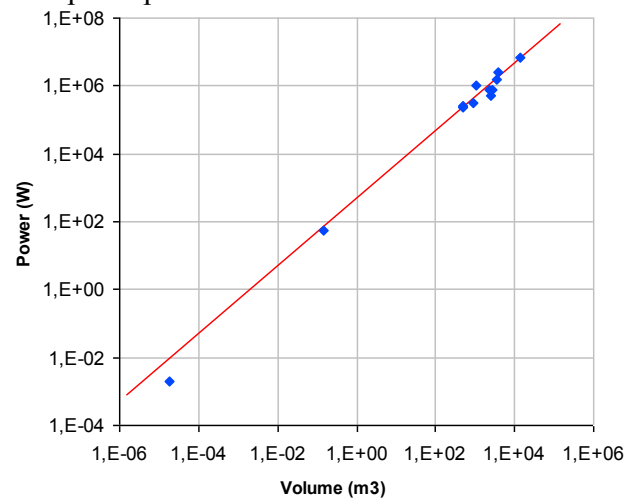

Fig. 8. Power per unit volume of various ocean wave harvesting devices.

\subsection{Energy harvesting with storage capacitance}

Sensors floating at ocean surface tend to use electric energy only at specific times to collect or transmit data. Therefore, the storage of the electrical energy transduced by the geophone in a capacitor via a diode bridge is considered here. Schottky diodes (BAT 41 Vishay) are used as they have a low forward voltage. Simulations are performed using the LTspice simulator with the circuit model of Fig.9. The variation of the calculated voltage across the storage capacitor with time is displayed in Fig. 10 for an input vibration velocity of $0.053 \mathrm{~m} . \mathrm{s}^{-1}$ at 
$5 \mathrm{~Hz}$ and different values of capacitance. It is found that the increase of the capacitance increases the charging time and decreases slightly the steady-state voltage across the capacitor. Thus, stored energy in the capacitor may be increased in steady-state by increasing the capacitance value if charging time is not an issue. Measurements are presented in Fig.11 for the same configuration. They are in good agreement with simulation for steady-state voltage across the capacitor (with some ripple because the filter capacitance is not large enough) and qualitative effect of capacitance increase. However, measured charging time are smaller than simulated ones by more than one order of magnitude.

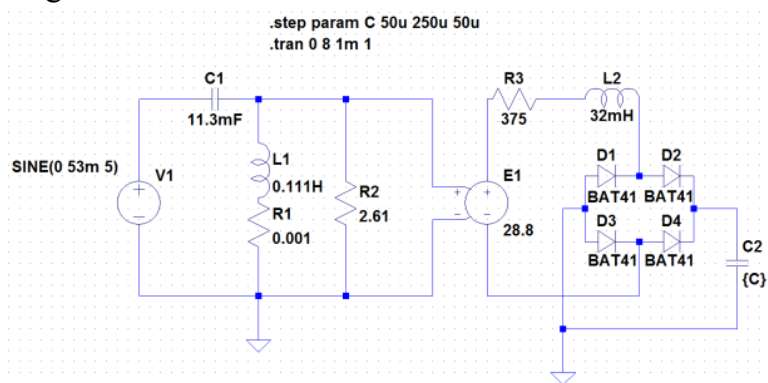

Fig. 9. Electrical circuit model of the geophone energy harvester using LT Spice.

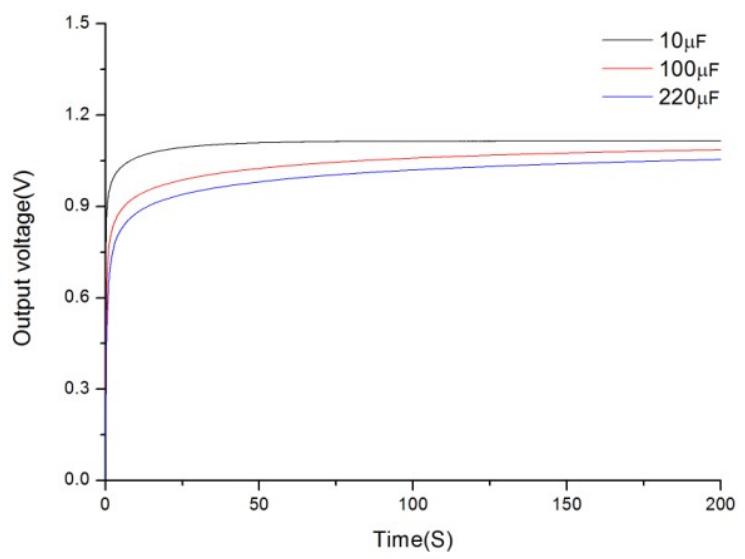

Fig. 10. Simulation of voltage of storage capacitor versus time for different capacitances.

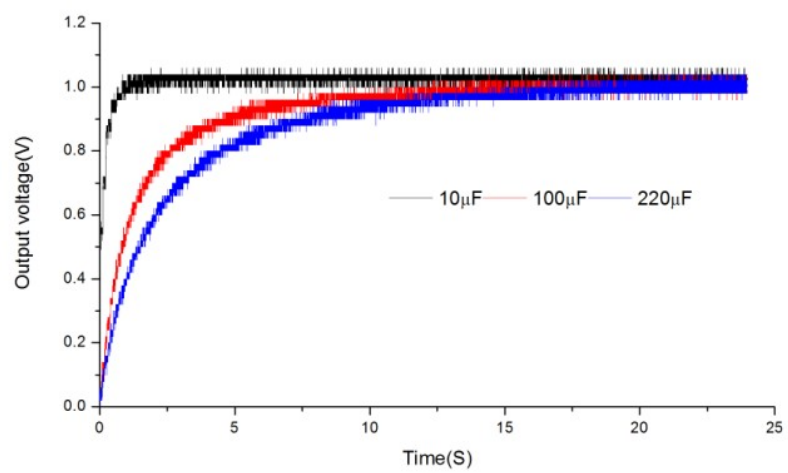

Fig. 11. Measurement of voltage of storage capacitor versus time for different capacitances.

\section{Conclusions}

Preliminary simulation and experimental results on a small-scale ocean wave harvester show that electrical power of approximately $0.1 \mathrm{~mW}$ may be harvested with a $20 \mathrm{~cm}^{3}$ geophone integrating a moving coil transducer. Similar computed and measured values are obtained for dissipated or stored electrical energy in the case of resistive or capacitive loads respectively. Large difference between simulated and measured time constants of capacitor charge emphasizes the need for a better model for the Schottky diodes. Harvesting power is currently limited by the displacement amplitude of the geophone. An optimised transducer allowing for a larger displacement could reach a power of 2-3 $\mathrm{mW}$.

This work was supported by the Chinese Scholarship Council.

\section{References}

1. L Christensen, E. Friis-Madsen, J. P. Kofoed, Proceedings of PowerGen Europe Conference, (2005)

2. M. Benoit, J.-F. Dhédin, G. Mattarolo, Conférence Institut Coriolis, (2010)

3. H. Lamb, Hydrodynamics (6th ed.), Cambridge University Press, (1994)

4. W. H. Munk, Proceedings of the 1st International Conference on Coastal Engineering. 1-4 (1945)

5. Y. Zhao, L. Wang, X. Yan, Proceedins of the $2^{\text {nd }}$ Conference on Electrical and Electronics: Techniques and Applications, 269-275 (2017)

6. L. Beranek, Acoustics, McGraw-Hill, (1954)

7. M. Bruneau, Manuel d'acoustique fondamentale, éditions Hermes, (1998)

8. S. Cheng, N. Wang, D.P. Arnold, J. Micromech. Microeng. 17, 2328-2335 (2007)

9. X. D. Xie, Q. Wang, N. Wu, J. Sound Vib. 333, 1421-1429 (2014) 